\title{
A quantum cascade laser-based Mach-Zehnder interferometer for chemical sensing employing molecular absorption and dispersion
}

\author{
Jakob Hayden $^{1}$ (i) $\cdot$ Stefan Hugger ${ }^{2} \cdot$ Frank Fuchs $^{2} \cdot$ Bernhard LendI $^{1}$
}

Received: 6 September 2017 / Accepted: 18 January 2018 / Published online: 27 January 2018

(c) The Author(s) 2018. This article is an open access publication

\begin{abstract}
We employ a novel spectroscopic setup based on an external cavity quantum cascade laser and a Mach-Zehnder interferometer to simultaneously record spectra of absorption and dispersion of liquid samples in the mid-infrared. We describe the theory underlying the interferometric measurement and discuss its implications for the experiment. The capability of simultaneously recording a refractive index and absorption spectrum is demonstrated for a sample of acetone in cyclohexane. The recording of absorption spectra is experimentally investigated in more detail to illustrate the method's capabilities as compared to direct absorption spectroscopy. We find that absorption signals are recorded with strongly suppressed background, but with smaller absolute sensitivity. A possibility of optimizing the setup's performance by unbalancing the interferometer is presented.
\end{abstract}

\section{Introduction}

Mid-infrared (Mid-IR) absorption spectroscopy of liquids finds a wide range of applications in chemical sensing due to its simplicity, speed and outstanding selectivity. More recently, quantum cascade lasers (QCL) were employed successfully to replace notoriously weak thermal emitters as Mid-IR sources. In particular, external cavity (EC) QCLs proved to be an ideal match for many liquid sensing applications owing to their large spectral tuning range of typically $200 \mathrm{~cm}^{-1}$ [1-9]. To date, the vast majority of measurements are performed using direct absorption spectroscopy, in which the laser beam's intensity is recorded after passing

This article is part of the topical collection "Mid-infrared and THz Laser Sources and Applications" guest edited by Wei Ren, Paolo De Natale and Gerard Wysocki.

Electronic supplementary material The online version of this article (https://doi.org/10.1007/s00340-018-6899-8) contains supplementary material, which is available to authorized users.

Bernhard Lendl

bernhard.lendl@tuwien.ac.at

1 Institute of Chemical Technologies and Analytics, Technische Universität Wien, Getreidemarkt 9/164, 1060 Vienna, Austria

2 Fraunhofer Institute for Applied Solid State Physics IAF, Tullastrasse 72, 79108 Freiburg, Germany through the sample or being totally reflected at its surface. Since this technique relies on recording small changes of the laser intensity, its sensitivity is ultimately limited by fluctuations of the laser power and noise from the detection system.

As of today, documented advantages of EC-QCL based systems over Fourier transform infrared spectrometers with thermal light sources are mainly increased ruggedness made possible by an enlarged optical path [10]. For example, recently an EC-QCL was successfully employed for the determination of protein secondary structures and protein folding in water using an optical path of $38 \mu \mathrm{m}$ instead of $8 \mu \mathrm{m}$ as required in FTIR spectrometry [10]. The so far achieved sensitivities of fully room temperature operated EC-QCL based systems compare well with those documented with high-end FTIR spectrometers which, however, employ liquid nitrogen cooled MCT detectors [11, 12].

For gas phase measurements, monolithic distributed feedback (DFB) QCLs have already been established as reliable light sources void of mechanical imperfections limiting the performance of EC-QCLs, albeit of a far reduced tuning range. Nevertheless, considering the narrow ro-vibrational transitions in small gas molecules, a range of new measurement approaches expanding on direct absorption spectroscopy have been demonstrated to feature important advantages in terms of ruggedness as well as sensitivity $[13,14]$. Some of these novel approaches exploit molecular dispersion, rather than absorption, to decouple the recorded quantity (phase or frequency) from the laser intensity $[15,16]$. 
For liquids, these developments are yet pending. Linewidths of tens of $\mathrm{cm}^{-1}$, the accordingly required lasers with tuning ranges beyond those achievable with DFB-QCLs, highly absorbing matrices and generally less well defined samples preclude a direct technology transfer from gas- to liquid spectroscopic sensing.

We present a novel, alternative approach to direct absorption spectroscopy for liquid samples that simultaneously and independently senses a sample's absorption and dispersion (refractive index). This is made possible by employing an interferometric principle of operation in a Mach-Zehnder type optical setup. Although single wavelength (integrated) Mach-Zehnder interferometers (MZI) are widely employed for (bio-)chemical sensing, especially in the visible spectral range $[17,18]$, a report on directly recording a liquid sample's absorption and dispersion spectrum in the Mid-IR using a MZI is, to the best of our knowledge, yet pending. The reader is, however, pointed to infrared spectroscopic ellipsometry [19] and dispersive Fourier transform spectroscopy $[20,21,22]$ that were used in (far-) infrared spectroscopic measurements of the complex dielectric function, mostly in reflection measurements.

We derive simple expressions relating the signals recorded in our setup to refractive index and absorption. Based on theory and experimental results we discuss distinct differences between direct absorption spectroscopy and our setup and how they can be exploited to extend the scope of QCL-based Mid-IR spectroscopy.

\section{Setup}

The setup is depicted schematically in Fig. 1. It is based on a free space MZI illuminated by an EC-QCL. A sampleand a reference liquid cell are introduced into the arms of the MZI, making the setup analogous to dispersive Fourier transform spectroscopy. The MZI is adjusted to equal optical path lengths in the two arms. To minimize fringing, all transparent optics, except for the liquid cells, are placed in the beam path in Brewster's angle and the light is $\mathrm{p}$-polarized, resulting in the rhombic shape of the interferometer.

The two interfering partial beams leaving the interferometer to the right in Fig. 1 (one from the reference arm and one from the sample arm of the interferometer) are recorded at a thermoelectrically cooled MCT detector, while a $\mathrm{BaF}_{2}$-window is tilted to adjust the phase $\varphi_{\text {rel }}$ between them to $\pi$, i.e. to best possible destructive interference. The window is twice as thick as the beam splitters which compensates for dispersion picked up by the reference (upper) beam in Fig. 1 within the beam splitters' substrate. With no or equal samples in the liquid cells, the partial beams' amplitudes are equal, resulting in full destructive interference and no signal from the MCT-detector. Equal amplitudes are

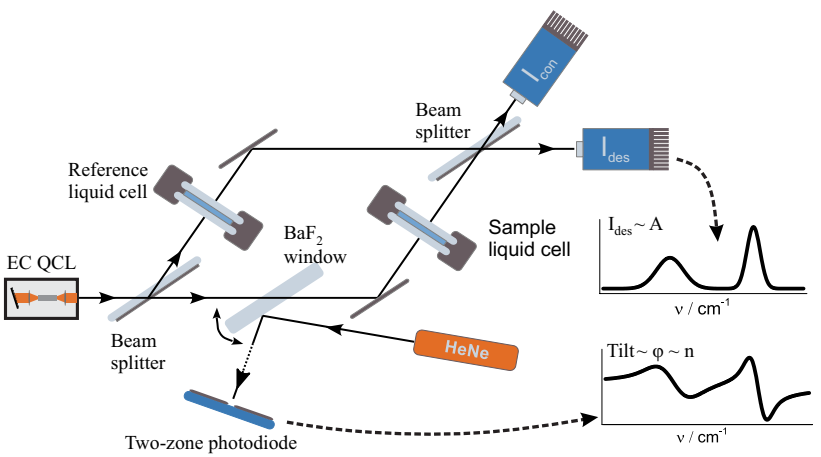

Fig. 1 Experimental setup. Light from the EC-QCL enters the MachZehnder-type interferometer through a beam splitter and passes the reference- or the sample liquid cell. The intensity $I_{\text {des }}$ of the destructively interfering fields leaving the MZI is measured by two mercury-cadmium-telluride detectors and is a measure of the sample's absorption. Destructive Interference is ensured by regulating $I_{\text {des }}$ to its minimum by tilting a $\mathrm{BaF}_{2}$ window in the sample beam path. The position sensitive photo diode facilitates the measurement of tilt, yielding the sample's refractive index

ensured by both beams being once transmitted and reflected at beam splitters of equal beam splitting ratios.

The basic principle of operation is as follows: When the sample liquid cell is filled with an absorbing species, the amplitudes of the interfering fields differ. Hence, destructive interference will be incomplete and a detectable signal is recorded that is a measure of the sample's absorption. At the same time, dispersion in the sample will cause a change in the relative phase of the two fields which needs to be compensated by the $\mathrm{BaF}_{2}$ window's tilt. By reading the tilt, the sample's refractive index is recorded. The tilt of the window is measured via the position of a reflected $\mathrm{HeNe}$-laser beam on a two zone photodiode. Optionally, the constructively interfering output of the MZI can be recorded by a second MCT detector and used to reference the destructively interfering signal to the laser power.

\section{Theory}

\subsection{Basic equations}

The signal $I_{\text {des }}$ recorded at the detector is modelled as the coherent superposition of two plane wave electric fields $E_{\mathrm{s}}$ and $E_{\text {ref }}$ of intensities $I_{\mathrm{s}}$ and $I_{\text {ref }}$, relative phase $\varphi_{\text {rel }}$ and equal frequency. $E_{\mathrm{s}}$ and $E_{\mathrm{ref}}$ are defined as the fields on the detector that were transmitted and reflected, respectively, at the first beam splitter of the MZI. This yields

$I_{\text {des }}=\left|E_{\mathrm{s}}+E_{\mathrm{ref}}\right|^{2}=I_{\mathrm{s}}+I_{\mathrm{ref}}+2 \sqrt{I_{\mathrm{s}} I_{\mathrm{ref}}} \cos \left(\varphi_{\mathrm{rel}}\right)$. 
During the experiment, the relative phase is set to $\pi$ by tilting the $\mathrm{BaF}_{2}$ window. In a symmetric interferometer with equal samples in both arms, $\varphi_{\text {rel }}$ does not change while scanning the wavelength and the window's tilt is constant. When the sample cell is loaded with a sample other than the reference, dispersion in the sample causes a spectrally varying additional phase $\varphi$ that needs to be actively compensated by tilting the window to set $\varphi_{\text {rel }}=\pi$. This phase $\varphi$, recorded via the window's tilt, is a measure of the sample's refractive index $n_{\mathrm{s}}$ as compared to the reference $n_{\text {ref }}$ and is given as

$\varphi=2 \pi \frac{d \times\left(n_{\mathrm{s}}-n_{\mathrm{ref}}\right)}{\lambda}$.

Herein, the film thickness $d$ was assumed equal for both cells. As can be seen from (2), the recorded phase is directly proportional to the sample's refractive index and hence, for diluted samples, its concentration $c$.

In the configuration described above, the intensities $I_{\mathrm{s}}$ and $I_{\text {ref }}$ are equal, except for absorption in the sample (decadic absorption is used throughout).

$I_{\mathrm{s}}=I_{\mathrm{ref}} 10^{-A}=I_{0} T_{\mathrm{ref}} R T 10^{-A}$

Herein, $I_{0}$ is the laser intensity, $T_{\text {ref }}$ the transmission of the reference and $R=1-T$ the reflectivity of the beam splitters. Combining (1) and (3) and setting $\varphi_{\text {rel }}=\pi$ yields

$I_{\text {des }}(A)=I_{\text {ref }} \times\left(1-10^{-A / 2}\right)^{2}$.

For calibration purposes using Beer's law it is useful to write out $A$ explicitly from (4).

$A=\varepsilon \times c \times d=-2 \log _{10}\left(1-\sqrt{\frac{I_{\text {des }}}{I_{\text {ref }}}}\right)$

\subsection{Discussion of interferometric signals}

When recording $I_{\text {des }}$ to measure concentrations or absorptions, some pronounced differences to direct absorption spectroscopy need to be taken into account. Most of them stem from the interferometric setup being sensitive to the amplitude of the transmitted E-field ('amplitude spectroscopy'), while direct absorption measures its power ('power spectroscopy') [22]. This behaviour is indicated by the square root of the fraction $I_{\text {des }} / I_{\text {con }}$ in the logarithm in (5) rather than the fraction of two intensities (as is the case in direct absorption spectroscopy). The differences between amplitude- and power spectroscopy can be seen in the graphs of $I_{\mathrm{s}}$ (direct absorption signal, compare (3)) and $I_{\text {des }}$ (Fig. 2) as a function of $A$ as well as from Taylor series expansion of $I_{\text {des }}$ in $A$ around $A_{0}=0$.

$I_{\mathrm{des}}=I_{\mathrm{ref}} \times\left[0+0 A+\frac{\ln (10)^{2} A^{2}}{4}-\frac{\ln (10)^{3} A^{3}}{8}+\cdots\right]$

One important feature of the interferometric signal is that it is background free, i.e. $I_{\text {des }}(0)=0$ (no constant

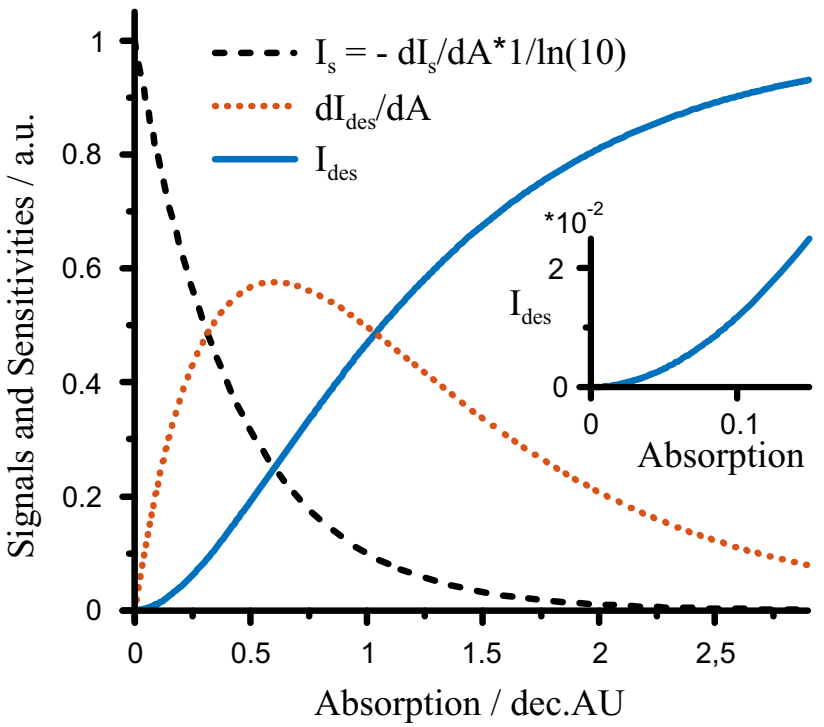

Fig. 2 Graphs of absorptive signals recorded in direct absorption spectroscopy $\left(I_{\mathrm{s}}\right)$ and the Mach-Zehnder interferometer $\left(I_{\mathrm{des}}\right)$ as well as their sensitivities $\mathrm{d} I / \mathrm{d} A$

term in (6)). However, as indicated by the vanishing linear term of (6), its sensitivity $\mathrm{d} I_{\mathrm{des}} / \mathrm{d} A$ is zero too. The sensitivity increases with $A$ until a maximum is reached at $A=2 \ln (2) / \ln (10) \approx 0.6$, with a shallow role off towards larger $A$. This behaviour is in strong contrast to the direct absorption signal $I_{\mathrm{s}}$, which has its largest magnitude (background) and largest sensitivity at $A=0$, decreasing with $A$.

Due to the vanishing sensitivity at small $A$ the MZI should in general not be operated with equally intense reference and sample beams. By increasing $I_{\text {ref }}$ relative to $I_{\mathrm{s}}$, the sensitivity can be strongly enhanced. This can be implemented by using beam splitters of different splitting ratios or-when dealing with samples featuring broadband background absorptions-by simply using a sample liquid cell of larger film thickness as compared to the reference. The latter approach potentially increases the method's sensitivity due to increased film thickness, but imbalances the optical path lengths in the interferometer, which requires additional care when interpreting the recorded spectra of $I_{\mathrm{des}}$ and $\varphi$. A generalized theory for unbalanced interferometers is derived in the Appendix.

For large absorptions $A>1$, the sensitivity of the interferometric signal is much larger than the quickly decreasing sensitivity $\mathrm{d} I_{\mathrm{s}} / \mathrm{d} A$ of the direct absorption signal. This makes the technique an interesting candidate when high concentrations need to be measured at good precision or for strongly absorbing solvents such as water. Also, the good sensitivity for large $A$ yields a significantly larger dynamic range of $I_{\text {des }}$ as compared to direct absorption spectroscopy.

Just like in conventional FTIR spectroscopy, $I_{\text {des }}$ needs to be referenced to the laser power and the absorption of the 
reference sample, i.e. to $I_{\text {ref }}$. In comparison to direct absorption spectroscopy, however, referencing is much less critical because the interferometer inherently references the sample beam to the reference beam. Drifting laser power solely scales $I_{\text {des }}$ (and retrieved $A$ and $c$ ), but does not add constant errors to it, as is the case for power spectroscopy. A detailed comparative discussion of LODs in the presence of detector noise, shot noise and laser intensity noise is given in Sect. 6.

The simultaneously recorded refractive index is independent from laser power and provides additional robustness towards laser power fluctuations. For diluted samples, refractive index spectra scale linearly with concentration and show highly characteristic features in the Mid-IR, making them a useful tool for chemical analysis.

\section{Experimental}

\subsection{Spectra acquisition and instrumentation}

The optical setup was described above. For all measurements presented herein, two close to identical beam splitters with a 50/50 splitting ratio were employed. The liquid cells' film thickness was $1.9 \mathrm{~mm}$. Uncoated $\mathrm{BaF}_{2}$ windows were employed as windows for the liquid cells. With the liquid cells filled with the same sample, the optical path length in the two arms was found to be equal on the order of the wavelength used. This was confirmed by scanning the wavelength over approximately $200 \mathrm{~cm}^{-1}$ without changing the $\mathrm{BaF}_{2}$-window's tilt and recording the destructive signal. The absence of spectral oscillations in the recorded interferometric signal indicates equal optical path lengths. The modulation efficiency $M$ of the interferometer, defined as the contrast between constructively and destructively interfering signals, $M=I_{\text {con }}(A=0) / I_{\text {des }}(A=0)$, exceeded $23 \mathrm{~dB}$ throughout the experiments (fringes from the liquid cells not taken into account). It was limited by slightly different intensities of the reference-and sample beam as well as imperfect overlapping of the beams at the detector.

To record spectra of $I_{\mathrm{des}}$ and $\varphi$, a simple program on a personal computer was used to tune the EC-QCL's wavelength across the desired range stepwise. At each step, the $\mathrm{BaF}_{2}$-window, mounted in a piezo-motor driven kinematic mount (Newport Agilis), was tilted in a closed loop to minimize the signal $I_{\text {des }}$ at the MCT detector. The closed loop differential controller was implemented in software to step the window's angle by a given value for a predefined number of times. Each step's direction was determined by the change in destructive signal upon the previous step to move towards smaller $I_{\mathrm{des}}$. The smallest recorded value of $I_{\mathrm{des}}$ as well as $I_{\text {con }}$ and its corresponding tilt-signal was stored.

The EC-QCL, developed and manufactured at Fraunhofer IAF Freiburg, provided a tuning range from 1080 to $1370 \mathrm{~cm}^{-1}$ in pulsed mode with an average power of $250 \mathrm{~mW}$ within a 120 -ns pulse at $1200 \mathrm{~cm}^{-1}$ [23]. Spectra were recorded by tuning the EC-QCL in steps of $0.4 \mathrm{~cm}^{-1}$ at a rate of $0.2 \mathrm{steps} / \mathrm{s}$. The acquisition speed was limited by the slow closed loop for tilting the window, especially the actuator, which was by no means optimized towards speed. $I_{\text {des }}$ as well as the attenuated second output of the interferometer, $I_{\text {con }}$, was measured by thermoelectrically cooled MCTdetectors (PVI-10.6, Vigo Systems S.A.) which were read out by a boxcar integrator at the laser repetition frequency of $9 \mathrm{kHz}$ (limited by the maximum frequency of the gated integrator) and averaged over 300 pulses. $I_{\text {con }}$ was used to reference $I_{\text {des }}$ to the laser power and the reference absorption spectrum by calculating $I_{\mathrm{des}} / I_{\mathrm{con}}$. This is only valid for small absorptions and sufficient to demonstrate the characteristics of the interferometric setup within the context of this work. For a rigorous calibration, a separately recorded spectrum of $I_{\text {ref }}$ must be used to calculate $A$ from $I_{\text {des }}$ using (5).

Reference absorption spectra were recorded on a Bruker Vertex 80v-FTIR spectrometer.

\subsection{Chemicals and samples}

Samples of acetone in cyclohexane at varying concentrations were prepared from reagent grade chemicals. Methanol added to some of the samples was of the same grade.

\section{Experimental results}

\subsection{Simultaneous measurement of absorption- and refractive index spectra}

The simultaneously measured spectra of $I_{\text {des }} / I_{\text {con }}$ and the $\mathrm{BaF}_{2}$ window's tilt of $2.4 \mathrm{mmol} / \mathrm{L}$ acetone in cyclohexane are depicted in Fig. 3. The spectra were smoothed using a Savitzky-Golay filter to remove fringes that could be assigned to the liquid cells. Inverse fast Fourier transformation of the raw spectra revealed multiple etalons of round trip optical path lengths between 4.5 and $7.5 \mathrm{~mm}$, well separated from the signal spanning out from DC to $0.2 \mathrm{~mm}$ (FWHM of studied absorption profile is $8 \mathrm{~cm}^{-1}$ ). The $\mathrm{BaF}_{2}$ window's tilt resembles the sigmoid anomalous dispersion profile of the refractive index. Kramers Kronig transformation yields an expected change in refractive index (peak to trough) of $\Delta n_{\text {max,min }} \approx 7 \times 10^{-5}$, corresponding to a phase difference of $6^{\circ}$, around the molecular resonance.

\subsection{Dilution series of acetone in cyclohexane}

Absorption spectra of different concentrations of acetone in cyclohexane were recorded. To demonstrate that no second detector is required to derive a reference for normalization, 


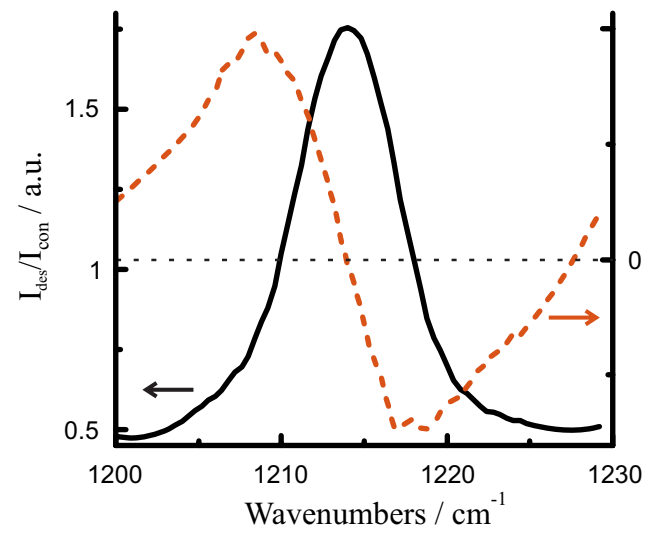

Fig. 3 Smoothed absorptive signal (solid line) and tilt of the $\mathrm{BaF}_{2}$ window (dashed line) of $2.4 \mathrm{mmol} / \mathrm{L}$ acetone in cyclohexane. Note that $I_{\text {des }}$ is normalized to a small fraction of $I_{\text {con }}$, explaining $I_{\mathrm{des}} / I_{\mathrm{con}}>1$

the interferometric spectra presented in Fig. 4 were normalized as $\left(I_{\mathrm{des}}-I_{\mathrm{des}}(c=0)\right) / I_{\mathrm{des}}(c=0) . I_{\mathrm{des}}(c=0)$ was recorded for pure cyclohexane in the sample- and reference position. Although this signal should be zero according to the theory derived above, due to limited modulation efficiency, $I_{\text {des }}(c=0)$ represents a background spectrum that was found to be a good measure for $I_{\text {ref }}$.

The normalized spectra of the recorded intensity $I_{\text {des }}$ in Fig. 4 qualitatively exhibit the expected behaviour of the interferometric signal discussed above with the sensitivity clearly increasing for larger concentrations, i.e. larger absorption $A$. After converting concentration to absorption via $A=c \times 0.0359 \mathrm{~L} / \mathrm{mmol}$, the parameters $a_{1}$ and $a_{2}$ of the function $I_{\mathrm{des}}=a_{1} \times\left(1+10^{-\left(A+a_{2}\right)}-2 \times 10^{-\left(A+a_{2}\right) / 2}\right)$ were fitted to the signal at the absorption maximum (Fig. 4, bottom). $a_{2}$ represents an offset absorption that resembles the small imbalance in the intensities of the reference- and sample beam.

\subsection{Increased reference intensity}

As discussed above, the absorptive signal exhibits its smallest sensitivity $\mathrm{d} I_{\text {des }} / \mathrm{d} A$ when the intensities of the reference and the sample beam are equal $(A=0)$ and increases as the two are unbalanced (increasing $A$, compare Fig. 2). To demonstrate the effect of unbalancing the signal and reference intensity, varying amounts of a spectrally flat absorber (methanol) were added to a sample of $3 \mathrm{mmol} / \mathrm{L}$ acetone in cyclohexane and their interferometric spectra were recorded with cyclohexane as a reference (Fig. 5, top left). The absorption spectrum of $3 \mathrm{mmol} / \mathrm{L}$ acetone in cyclohexane, recorded on an FTIR spectrometer, was used to replace the wavenumber axis of

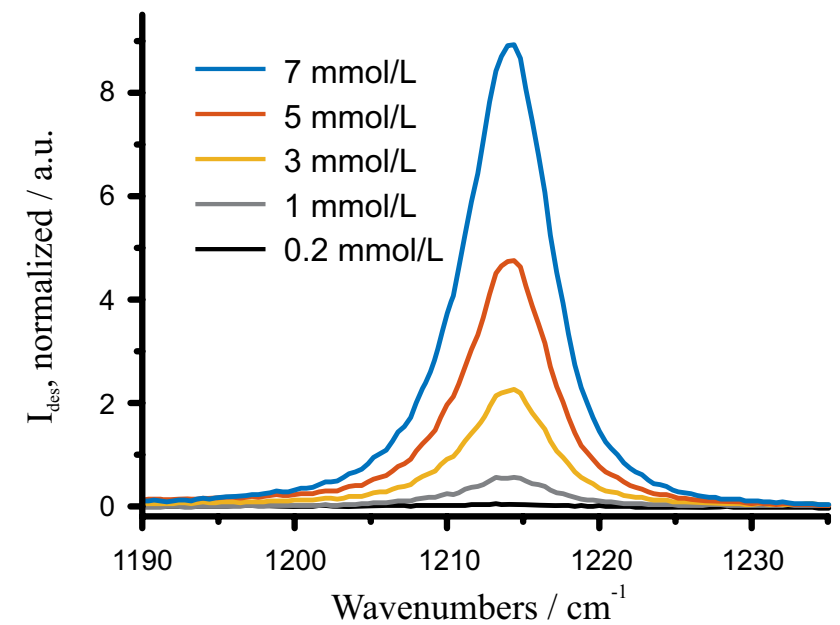

Fit:

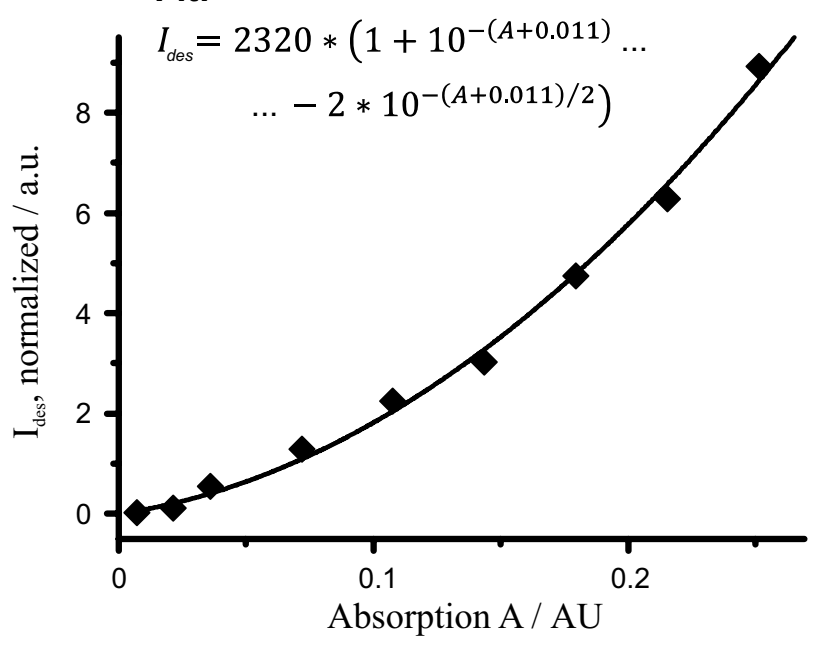

Fig. 4 Top; absorptive signal $\left(I_{\mathrm{des}}-I_{\mathrm{des}}(c=0)\right) / I_{\mathrm{des}}(c=0)$ for varying concentrations of acetone in cyclohexane. Bottom; absorptive signal at absorption maximum $\left(1214 \mathrm{~cm}^{-1}\right)$ against peak absorption $A=c \times 0.0359 \mathrm{~L} / \mathrm{mmol}$

the interferometric spectra by an absorption axis (compare Fig. 5). The so derived curves of $I_{\text {des }} / I_{\text {con }}$ versus $A$ were analysed by fitting second-order polynomials to them. The fitted coefficients are given in Table 1 . The coefficient $K_{1}$, representing the sensitivity of the absorptive signals for small $A$, is increased by a factor of seven by reducing the intensity of the sample beam by 11 percent. At the same time, the background-signal $\left(K_{0}\right)$ is increased by a factor of five, giving rise to a trade-off between background intensity and sensitivity. As discussed in detail in Sect. 6, this trade-off must be made based on the sources of noise in the system.

As discussed above, the noticeable offset of $I_{\mathrm{des}} / I_{\mathrm{con}}$ for $I_{\mathrm{s}}=I_{\text {ref }}$ and $A=0$ in Fig. 5 arises from the limited modulation efficiency $M$ and the slightly differing intensities in the two interferometric arms (Fig. 7). 

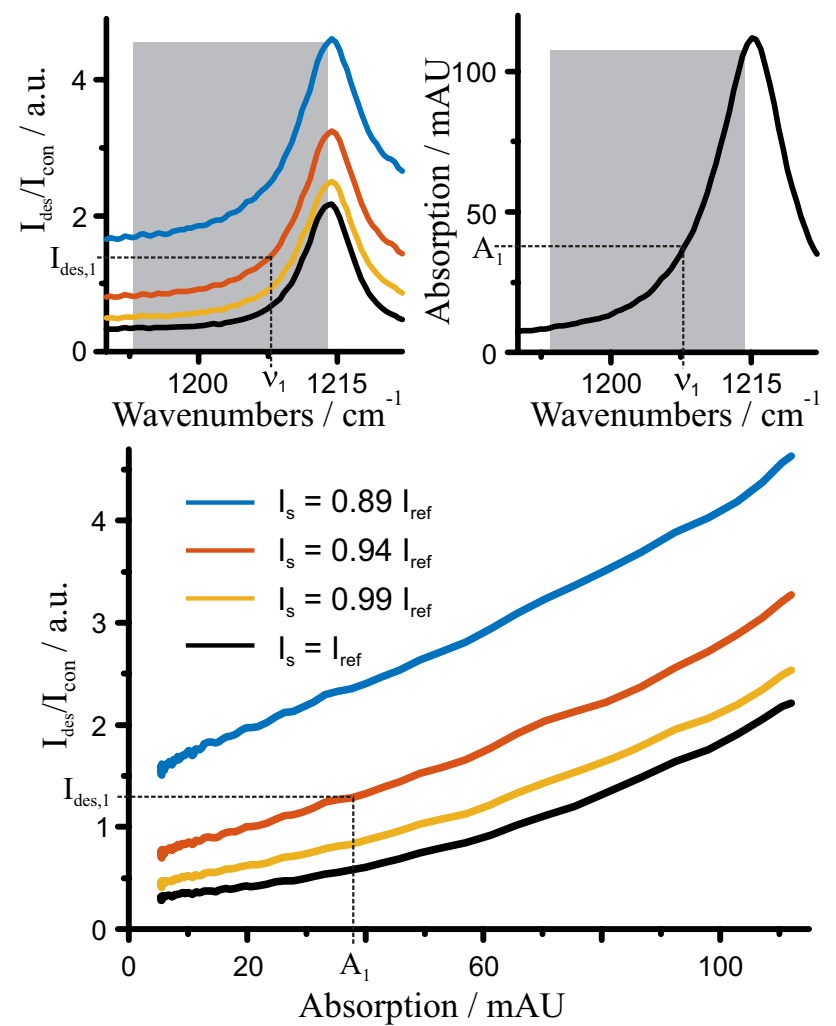

Fig. 5 Top, left: interferometric spectra of $3 \mathrm{mmol} / \mathrm{L}$ acetone in cyclohexane for decreasing intensities $I_{\mathrm{s}}$ in the sample arm of the interferometer. Top, right: absorption spectrum of the same sample from an FTIR spectrometer used to assign a value of absorption to any wavenumber within the range indicated by the grey area (e.g. $A_{1}$ for $\nu_{1}$ ). Bottom: normalized destructive signal versus sample absorption for different intensities $I_{\mathrm{s}}$ and $I_{\text {ref }}$

Table 1 Fitted parameters of $I_{\text {des }} / I_{\text {con }}=K_{0}+K_{1} A+K_{2} A^{2}$ for the curves shown in Fig. 5

\begin{tabular}{lllr}
\hline$I_{\mathrm{s}} / I_{\text {ref }}$ & $K_{0}$ & $K_{1}$ & \multicolumn{1}{c}{$K_{2}$} \\
\hline 1 & 0.30 & 3.0 & 130 \\
0.99 & 0.42 & 8.2 & 97 \\
0.94 & 0.70 & 13 & 86 \\
0.89 & 1.5 & 21 & 59 \\
\hline
\end{tabular}

\section{Modelling limits of detection}

Experimental shortcomings of the current setup (fringes, poor phase adjustment, limited ruggedness) prohibit a direct experimental assessment of figures of merit as compared to established direct absorption spectroscopy (e.g. FTIR). Here, we give a comparative discussion based on the equations presented above and a noise model including shot noise, detector noise and power noise of the light source. For the Mach-Zehnder interferometer, noise associated with the interferometric phase is considered negligible, as can be expected for a rugged design and

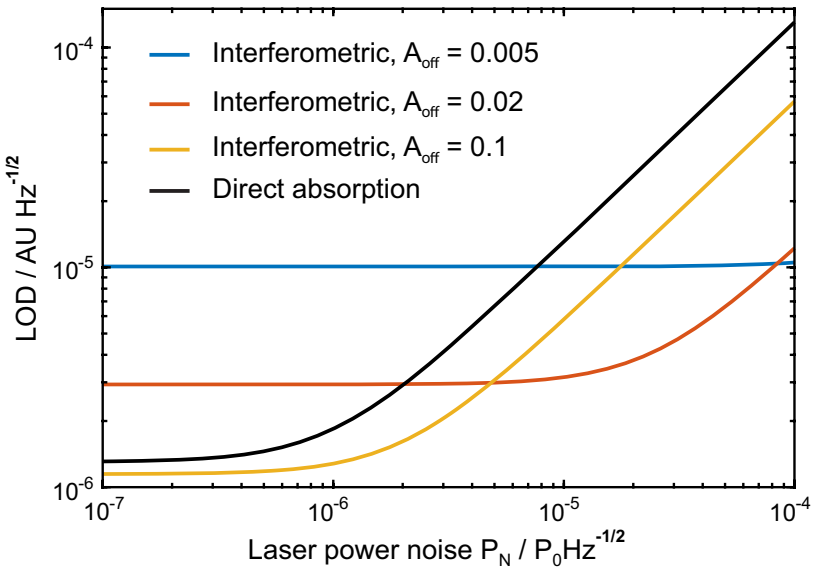

Fig. 6 Estimated LODs of the interferometric-and a direct absorption measurement. For details, see text

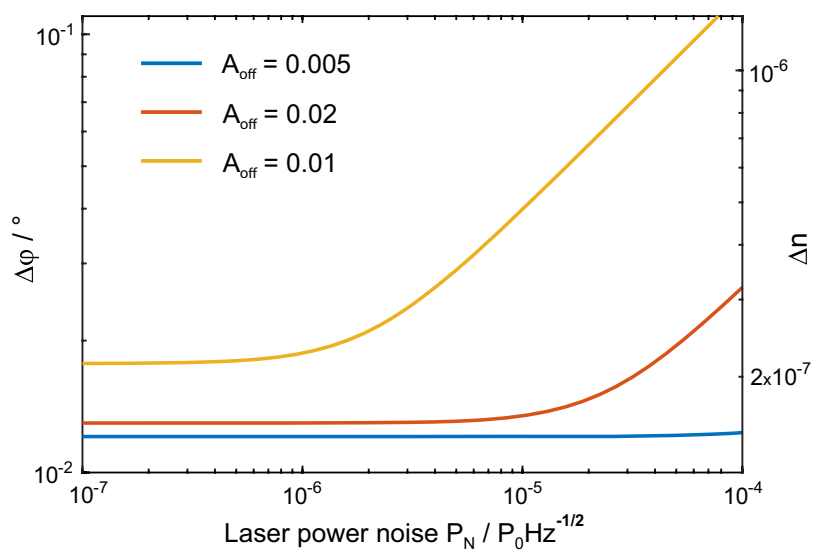

Fig. 7 Estimated uncertainties of an interferometric phase and refractive index measurement of $1 \mathrm{~Hz}$ bandwidth. Assumptions equal to Fig. 6, see text

well-optimized feedback loop that keeps the phase at $\pi$. For both, direct absorption and interferometric measurements, fringes are indistinguishable from absorption (and dispersion) in the equations and effect the measurements equally.

The noise equivalent power (NEP), given in $\mathrm{WHz}^{-1 / 2}$, overlying the recorded signals, is modelled as

$\mathrm{NEP}=\sqrt{\mathrm{NEP}_{\text {det }}^{2}+P_{\text {shot }}^{2}+P_{\mathrm{PN}}^{2}}$

Herein, $P_{\text {shot }}, \mathrm{NEP}_{\operatorname{det}}$ and $P_{\mathrm{PN}}$ are shot noise, the noise equivalent power of the detector and power noise from the laser (or other light source), respectively. Shot noise is given as

$P_{\text {shot }}=\sqrt{h \nu P_{\text {sig }}(A=0)}$ 
with $h$ representing Planck's constant and $\nu$ the optical frequency. The power of the detected light $P_{\text {sig }}$ is given by (compare (4))

$P_{\text {sig,int }}=P_{0} R T\left(1-10^{-\frac{A+A_{\text {off }}}{2}}\right)^{2}$

for the interferometric signal and by

$$
P_{\text {sig, dir }}=P_{0} 10^{-A}
$$

for direct absorption, with $P_{0}$ representing the laser power. $A_{\text {off }}$ represents an intentionally introduced imbalance between the intensities of the destructively interfering fields (compare Sect. 5.3). $P_{\mathrm{PN}}$ is derived by considering the effect of normally distributed laser power noise of power $P_{\mathrm{N}}$ on the recorded signals $P_{\text {sig }}$ for $A=0$.

$P_{\mathrm{PN}, \mathrm{dir}}=P_{\mathrm{N}}$

$P_{\mathrm{PN}, \text { int }}=P_{\mathrm{N}} R T\left(1-10^{-\frac{A_{\mathrm{off}}}{2}}\right)^{2}$

$\mathrm{NEP}_{\text {det }}$, often specified inversely as detectivity $D^{*}$, usually scales with the power of light to be detected. Typical $\mathrm{NEP}_{\text {det }}$ for Mid-IR detectors are on the order of $10^{-6} \mathrm{~Hz}^{-1 / 2}$ of the saturation power. Assuming an amplification chosen to match the highest expected signals, $N E P_{\text {det }}$ is given as

$$
\begin{aligned}
& \mathrm{NEP}_{\text {det,dir }}=a \times P_{\text {sig,dir }}(A=0)=a P_{0} \\
& \mathrm{NEP}_{\text {det,int }}=a \times P_{\text {sig, int }}\left(A_{\text {max }}\right) .
\end{aligned}
$$

From here on, we assume $A_{\max }=0.2$ (corresponding to $P_{\text {sig,int }}=10^{-2} P_{0}$ ) for the strongest interferometric signal. $a$ is assumed $10^{-6} \mathrm{~Hz}^{-1 / 2}$.

To calculate limits of detection (LOD), we employ

$$
\mathrm{LOD}=3 \times \frac{\mathrm{NEP}}{\mathrm{d} P_{\text {sig }} / \mathrm{d} A(A=0)}
$$

The sensitivities $\mathrm{d} P_{\text {sig }} / \mathrm{d} A$ read

$\frac{\mathrm{d} P_{\text {sig,int }}}{\mathrm{d} A}(A)=P_{0} \ln (10) R T \times\left(10^{-\frac{A+A_{\text {off }}}{2}}-10^{-\left(A+A_{\text {off }}\right)}\right)$

$\frac{\mathrm{d} P_{\text {sig, dir }}}{\mathrm{d} A}(A)=-P_{0} \ln (10) 10^{-A}$.

The so estimated LOD of the interferometric absorption measurement for three different offset absorptions $A_{\text {off }}$ as well as the LOD of the direct absorption measurement are plotted in Fig. 6 against the laser intensity noise $P_{\mathrm{N}}$. For small $P_{\mathrm{N}}$, signals are detector noise limited and independent from $P_{\mathrm{N}}$. With an offset absorption $A_{\text {off }}=0.1$, the interferometric measurement yields slightly smaller LODs due to the reduced detector noise associated with the reduced dynamic range of the detector. As $P_{\mathrm{N}}$ increases, the interferometric measurement yields smaller LODs. This is due to the interferometric referencing of the light from the sample arm of the interferometer to the reference arm, which cancels common mode noise. As $A_{\text {off }}$ increases, the increased sensitivity $\mathrm{d} P_{\text {sig,int }} / \mathrm{d} A$ yields lower LODs in the detector noise limited regime, but higher LODs for increased laser power noise. This is explained by incomplete destructive interference in the unbalanced interferometer and hence only partial removal of power noise.

Assuming no additional noise sources for the interferometric phase, LODs for the refractive index can be estimated based on the noise floor of the signal

$$
P_{\text {sig, int }}(\varphi)=P_{0} R T\left[1+10^{-\left(A+A_{\text {off }}\right)}-2 \times 10^{-\frac{A+A_{\text {off }}}{2}} \cos (\varphi)\right],
$$

since this signal's minimum with respect to $\varphi$ defines the refractive index (see (2)). An estimate of LODs equivalent to (15) using the sensitivity $\mathrm{d} P_{\text {sig,int }} / \mathrm{d} \varphi$ is prohibited by the vanishing sensitivity at $\varphi=0$. Instead, we propagate the error NEP of the $P_{\text {sig,int }}$ measurement to the measurement of phase by solving NEP $=P_{\text {sig, int }}(\Delta \varphi)-P_{\text {sig,int }}(0)$ for $\Delta \varphi$ setting $A=0$.

$\Delta \varphi=\arccos \left(1-\frac{\mathrm{NEP} \times \sqrt{B W}}{2 P_{0} R T \times 10^{-A_{\mathrm{off}} / 2}}\right)$

The measurement uncertainty $\Delta \varphi$ and resulting $\Delta \mathrm{n}$ are plotted in Fig. 7 against laser power noise $P_{\mathrm{N}}$ using the same parameters for NEP as in Fig. 6, a bandwidth BW of $1 \mathrm{~Hz}$, a liquid film thickness of $1.9 \mathrm{~mm}$ and a wavelength of $8.3 \mu \mathrm{m}$. For a comparison, refractive index and phase can be referenced to absorption using Kramers-Kronig transformation and assuming an isolated Gaussian absorption profile of peak absorbance $A$. For this profile, Kramers-Kronig transformation yields an anomalous dispersion profile spanning, from top to bottom, $n_{\max }-n_{\min }=A / d \times 1.86 \times 10^{-3} \mathrm{~mm}^{-1}$, or $\varphi_{\max }-\varphi_{\min }=A \times 80^{\circ} / A U$. For the Mach-Zehnder interferometer, this comparison yields a much better performance of absorptive measurements, which is a result of the vanishing sensitivity $\mathrm{d} P_{\text {sig,int }} / \mathrm{d} \varphi$ at $\varphi=0$.

\section{Conclusions}

In this contribution, we introduced an interferometric setup that facilitates the simultaneous measurement of absorption and refractive index spectra of liquid samples. We discussed the theory relating the recorded signals to absorption and dispersion and presented proof of principle measurements supporting theoretical predictions.

For the absorptive signal, the theoretical considerations and experimental data demonstrate important differences to 
direct absorption spectroscopy. Spectra are recorded close to background free and are inherently referenced to the sample (e.g. solvent or matrix) introduced into the reference position. Although the sensitivity, i.e. change of recorded signal with absorption or concentration, is reduced as compared to direct absorption spectroscopy, this does not imply higher limits of detection (LOD). The latter depend on the noise floor, which, assuming the same sources of noise, is very different for the two techniques. While the reduced sensitivity (smaller signals) makes interferometric absorption measurements more prone to detector noise, they are much less susceptible to intensity noise due to the reduced levels of background intensity at the detector. This is of particular interest for external cavity laser based spectroscopy, since these lasers are known for intensity noise associated with mode hops [10, 24]. A trade-off between sensitivity and immunity to intensity noise can be made by unbalancing the intensities of the reference and sample beam.

So far, an experimental investigation of these interesting properties was hampered by fringes in the sample cells as well as the slow actuator and feedback loop for the phase adjustment. Using wedged and/or anti reflection-coated windows could reduce the fringes. Adjustment of the phase can be greatly speeded up by using one of the interferometer's mirrors on a piezo element as the phase actuator [25].

Acknowledgements Open access funding provided by TU Wien (TUW). The authors want to thank QuantaRed Technologies for financial support, O. Ambacher and J. Wagner for support of the work at Fraunhofer IAF, Klaus Schwarz from Fraunhofer IAF for technical support and Christian Schilling for the design and fabrication of the beamsplitters at Fraunhofer IAF. Parts of this work were performed within the Competence Centre ASSIC-Austrian Smart Systems Research Center, co-funded by the Federal Ministries of Transport, Innovation and Technology (bmvit) and Science, Research and Economy (bmwfw) and the Federal Provinces of Carinthia and Styria within the COMETCompetence Centers for Excellent Technologies Programme.

Open Access This article is distributed under the terms of the Creative Commons Attribution 4.0 International License (http://creativeco mmons.org/licenses/by/4.0/), which permits unrestricted use, distribution, and reproduction in any medium, provided you give appropriate credit to the original author(s) and the source, provide a link to the Creative Commons license, and indicate if changes were made.

\section{Appendix: Generalized theory}

The interferometer can be imbalanced in two ways. The intensities $I_{\text {ref }}$ and $I_{\mathrm{s}}$ can differ (compare Sect. 5.3) or the film thickness of the liquid cells can be unequal.

The former situation can be easily described by the equations derived above by describing the difference between $I_{\text {ref }}$ and $I_{\mathrm{S}}$ via an offset absorption $A_{0}=\log \left(I_{\text {ref }} / I_{\mathrm{s}}\right)$, which modifies (3) to
$I_{\mathrm{s}}=I_{\text {ref }} \times 10^{-\left(A+A_{0}\right)}$

$A_{0}$ can be a constant or vary spectrally, depending on the mechanism creating the imbalance of intensities.

Unequal film thicknesses $d_{\text {ref }} \neq d_{\mathrm{s}}$ result in differing intensities of $I_{\mathrm{s}}$ and $I_{\text {ref }}$ because of absorption by the matrix and differing optical path lengths in the two liquid films. Increased matrix absorption enters the equations above just like $A_{0}$ in (20) and is given as $A_{\text {matrix }}=\alpha_{\text {matrix }} \times\left(d_{\mathrm{s}}-d_{\text {ref }}\right)$, with $\alpha$ being the decadic absorption coefficient. The optical path lengths are given as

$l_{\text {opt,s,ref }}=n_{\mathrm{s}, \text { ref }} \times d_{\mathrm{s}, \text { ref }}$

The difference between the two path lengths yields the phase $\varphi$ (compare (2)) as

$\varphi=\frac{2 \pi}{\lambda} \times\left[n_{\text {ref }}\left(d_{\mathrm{s}}-d_{\text {ref }}\right)+\left(n_{\mathrm{s}}-n_{\text {ref }}\right) d_{\mathrm{s}}\right]$

The extended form of (22) illustrates that $\varphi$ is composed of a background spectrum resembling the refractive index of the reference and a sample spectrum.

\section{References}

1. M. Brandstetter, B. Lendl, Sensors Actuators B Chem. 170, 189 (2011)

2. A. Schwaighofer, M. Brandstetter, B. Lendl, Chem. Soc. Rev. (2017)

3. A. Lambrecht, M. Pfeifer, W. Konz, J. Herbst, F. Axtmann, Analyst. 139, 2070 (2014)

4. V.A. Lórenz-Fonfría, B.J. Schultz, T. Resler, R. Schlesinger, C. Bamann, E. Bamberg, J. Heberle, J. Am. Chem. Soc. 137, 1850 (2015)

5. B.J. Schultz, H. Mohrmann, V.A. Lorenz-Fonfria, J. Heberle, Spectrochim. Acta Part A Mol. Biomol. Spectrosc. 188, 666 (2018)

6. A. Rüther, M. Pfeifer, V.A. Lórenz-Fonfría, S. Lüdeke, Chirality. 26, 490-496 (2014)

7. J. Haas, R. Stach, M. Sieger, Z. Gashi, M. Godejohann, B. Mizaikoff, Anal. Methods. 8, 6602 (2016)

8. M.L. Donten, S. Hassan, A. Popp, J. Halter, K. Hauser, P. Hamm, J. Phys. Chem. B. 119, 1425 (2015)

9. A. Popp, D. Scheerer, H. Chi, T.A. Keiderling, K. Hauser, ChemPhysChem. 17, 1273 (2016)

10. M.R. Alcaráz, A. Schwaighofer, C. Kristament, G. Ramer, M. Brandstetter, H. Goicoechea, B. Lendl, Anal. Chem. 87, 6980 (2015)

11. M. Brandstetter, T. Sumalowitsch, A. Genner, A.E. Posch, C. Herwig, A. Drolz, V. Fuhrmann, T. Perkmann, B. Lendl, Analyst. 138, 4022 (2013)

12. A. Schwaighofer, M.R. Alcaráz, C. Araman, H. Goicoechea, B. Lendl, Sci. Rep. 6, 33556 (2016)

13. I. Galli, S. Bartalini, R. Ballerini, M. Barucci, P. Cancio, M. De Pas, G. Giusfredi, D. Mazzotti, N. Akikusa, P. De Natale, Optica. 3, 385 (2016)

14. M.S. Taubman, T.L. Myers, B.D. Cannon, R.M. Williams, Spectrochim. Acta Part A Mol. Biomol. Spectrosc. 60, 3457 (2004)

15. G. Wysocki, D. Weidmann, Opt. Express. 18, 26123 (2010) 
16. P. Martín-Mateos, J. Hayden, P. Acedo, B. Lendl, Anal. Chem. 89, 5916 (2017)

17. M. Sieger, F. Ballu, X. Wang, S. Kim, L. Leidner, G. Gauglitz, B. Mizaikoff, Anal. Chem. 85, 3050 (2013)

18. P. Kozma, F. Kehl, E. Ehrentreich-Förster, C. Stamm, F.F. Bier, Biosens. Bioelectron. 58, 287 (2014)

19. K. Hinrichs, M. Gensch, N. Esser, Appl. Spectrosc. 59, 272A (2005)

20. J. R. Birch, Mikrochim. Acta 93, 105 (1987)

21. A.E. Martin, in Infrared Interferom. Spectrometers, ed. by J. R. Durig ed. (Elsevier, 1980), pp. 201-216
22 R.J. BELL, in Introd. Fourier Transform Spectrosc. (Academic Press, 1972), pp. 78-107

23 J. Wagner, R. Ostendorf, J. Grahmann, A. Merten, S. Hugger, J.-P. Jarvis, F. Fuchs, D. Boskovic, H. Schenk, in Proc. SPIE (2015), p. 937012

24 G. Wysocki, R.F. Curl, F.K. Tittel, R. Maulini, J.M. Bulliard, J. Faist, Appl. Phys. B. 81, 769 (2005)

25 C.C. Davis, Nucl. Phys. B Proc. Suppl. 6, 290 (1989) 